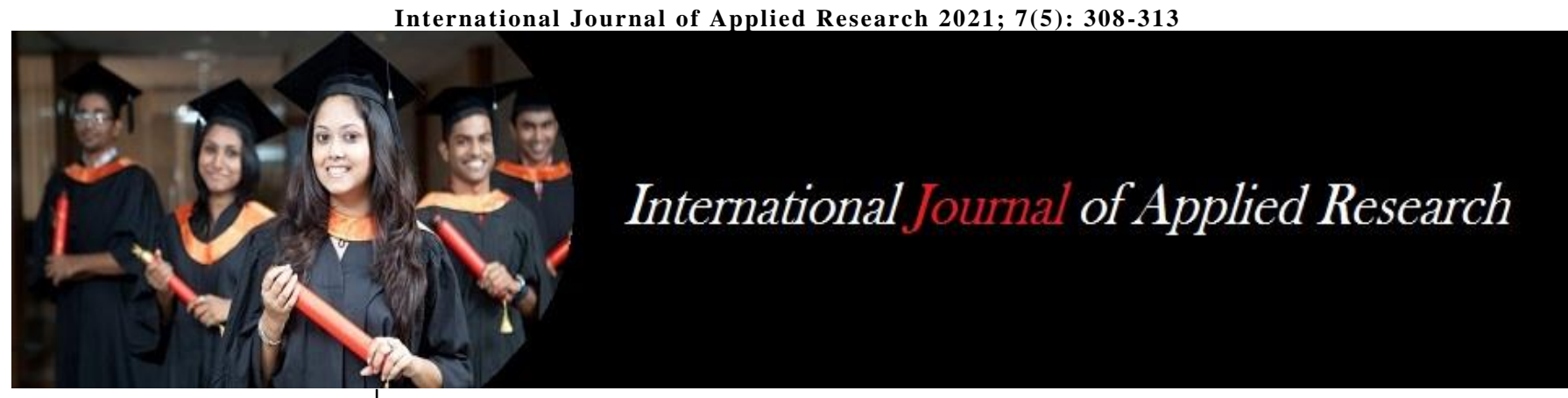

ISSN Print: 2394-7500

ISSN Online: 2394-5869

Impact Factor: 8.4

IJAR 2021; 7(5): 308-313

www.allresearchjournal.com

Received: 10-03-2021

Accepted: 12-04-202

\section{Pranav Shetty}

Department of Computer

Science, All India Shri Shivaji

Memorial Society's College of

Engineering Savitribai Phule

Pune University, Pune,

Maharashtra, India

Suraj Singh

Department of Computer

Science, All India Shri Shivaji

Memorial Society's College of

Engineering Savitribai Phule

Pune University, Pune,

Maharashtra, India

Rasvi Jambhulkar

Department of Computer

Science, All India Shri Shivaji

Memorial Society's College of

Engineering Savitribai Phule

Pune University, Pune,

Maharashtra, India

\section{Kajal Sheth}

Department of Computer

Science, All India Shri Shivaji

Memorial Society's College of

Engineering Savitribai Phule

Pune University, Pune,

Maharashtra, India

Deepali Ujlambkar

Professor Department of

Computer Science, All India

Shri Shivaji Memorial Society's

College of Engineering

Savitribai Phule Pune

University, Pune,

Maharashtra, India

Corresponding Author:

Pranav Shetty

Department of Computer

Science, All India Shri Shivaji

Memorial Society's College of

Engineering Savitribai Phule

Pune University, Pune,

Maharashtra, India

\section{Detection of brain tumor using $\mathrm{CNN}$ and ML}

\author{
Pranav Shetty, Suraj Singh, Rasvi Jambhulkar, Kajal Sheth and Deepali \\ Ujlambkar
}

DOI: https://doi.org/10.22271/allresearch.2021.v7.i5e.8585

\begin{abstract}
An automated neurological disorder identification system that uses computer vision on magnetic resonance imaging to locate brain tumors (MRI). The most common and dangerous form of brain cancer is gliomas. Gliomas are tumors that, at their most advanced stage, result in a much shorter life span. Preparing for therapy is an important step in maintaining a better quality of life for oncology patients. Magnetic resonance imaging (MRI) is a technique for examining the structures and components of the human body as well as for medical diagnosis, determining the stage of disease, and monitoring without the use of ionizing radiation. The significant spatial and structural changeability of brain tumors complicates segmentation. As a result, an automatic and consistent segmentation technique is used, based on Convolutional Neural Networks (CNN). Because of the relatively low number of network weights, the use of small kernels enables the development of a deeper architecture, with such a positive effect on over-fitting. It also explores the use of intensity normalization as a preprocessing phase, which is not widely used in segmentation techniques based on the Convolution Neural Network, but has been shown to be efficient in segmenting brain tumors using Magnetic Resonance Imaging (MRI) in conjunction with data augmentation.
\end{abstract}

Keywords: deep learning, brain tumors, convolutional neural networks $(\mathrm{CNN})$, magnetic resonance imaging (MRI)

\section{Introduction}

The human brain is a highly developed and diverse organ made up of extremely spongy and soft tissues. It is widely regarded as the human body's central processing device. Our brain helps us to transmit our expressions, execute our actions, and share beliefs, thoughts, and feelings. In such altered conditions, brain tissue development is unregulated. This abnormal rise in tissue density is referred to as a lump, and if it occurs within the brain, it is referred to as a brain tumor. Tumors appear to form new arteries in the blood. Malignant tumor diagnosis becomes more complex in large tumors. CSF (Cerebral Spinal Fluid) is normally affected by a brain tumor. CSF leakage can cause life-threatening conditions such as meningitis, brain infections, and stroke. As a result, early detection and proper diagnosis are needed.

The intended machine identifies the tumor and its shape using computer technology such as image recognition; in general, it is a framework for interpreting and processing recorded images in digital format for detailed data such as color and resolution. The concepts of image processing and MRI were used to develop a scan-based imaging technique for the detection and screening of brain tumors (Magnetic Resonance Imaging). This procedure is not only limited to the detection of tumors in the brain but it may also be used to scan the whole internal anatomy of the human body to detect any tumor.

\section{Existing System}

Magnetic resonance imaging (MRI) is the imaging modality of choice for brain tumor research. A single MRI examination can provide a plethora of data and details. When a radiologist has to make a diagnosis, he or she is faced with a larger number of sources of evidence but less testing instruments [1]. We suggest an automated data-driven tumor recognition approach in which both localization (segmentation) and characterization are used in the identification (signature) process. 
Several attempts have been made to analyze multiparameter quantitative MRI data to measure the information content of the affected area of the organ, but they have been much less influential than in standard MRI research ${ }^{[2]}$. They usually consist of two phases: localization and characterization, whose variability and precision can be managed in two ways: by automating ROI collection and standardizing quantitative feature extraction, respectively. The majority of strategies rely on one of the other perspectives: segmentation approaches are typical segmentation approaches focused on a few standard MRI maps, while a preliminary manual ROI delineation is devoted to more advanced feature extraction techniques.

The size of the brain tumor is determined using image processing techniques. The effective use of $\mathrm{K}$ - means and Fuzzy C- Means to predict the area and stage of a brain tumor overcomes the disadvantages of thresholding and region increasing algorithms. The Fuzzy C-Means algorithm uses the performance of the K-Means algorithm as feedback, resulting in tumor edge precision. This shows how fuzzy logic and neural networks can be used to diagnose and classify brain tumors. The latent facts are analyzed, extracted, and converted using the Brain Tumor methodology, which produces Devising Classifiers software artefacts for creating hierarchical structures like the Integrated Framework for Analysis and Classification of Brain Tumor ${ }^{[5]}$. The method is only useful on tumors that are similar to each other, and it is incapable of dealing with new types of tumors. As a result, we've come to the conclusion that, in order to improve tumor detection accuracy, we need to train the device with a variety of tumor types.

Other frameworks, such as ANN, have used various deep learning neural networks to produce reliable outcomes, but those schemes and systems needed a lot of hardware computation, which resulted in slower yields ${ }^{[6]}$.

\section{Proposed System}

The initiative presents us with several grounds to introduce trained radiologists' negligence. It provides continuous control over the propagation of a disease at all times, but the cost can be high as a result; however, it gives us accuracy in brain tumor prediction and also a strong suggestion of MRI. Our system's architecture is based on client- server applications. The app will accept an MRI as input and will output a comprehensive MRI result. To use this application, the user must include basic information such as his name, gender, age, email address, and MRI ${ }^{[1]}$. The machine will provide a performance that includes the lesion and the tumor's presumed diagnosis.

\section{Methodology of the system \\ 4.1 Image Thresholding}

The simplest method of thresholding is to set an intensity value as a threshold number, with values below it being 0 (black) and those above it becoming 1. (white). If $\mathrm{T}$ is the global threshold of image $f(x, y)$ and the $g(x, y)$ is the thresholding image, then:

$$
g(x, y)=\left\{\begin{array}{lr}
1, & \text { if } \quad f(x, y) \geq T \\
0, & \text { otherwise }
\end{array}\right.
$$

Fig 1: Image Thresholding Range

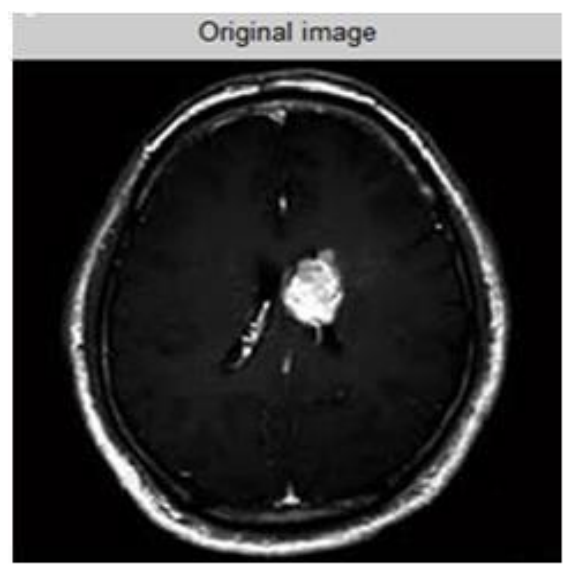

Fig 2: Image before Thresholding

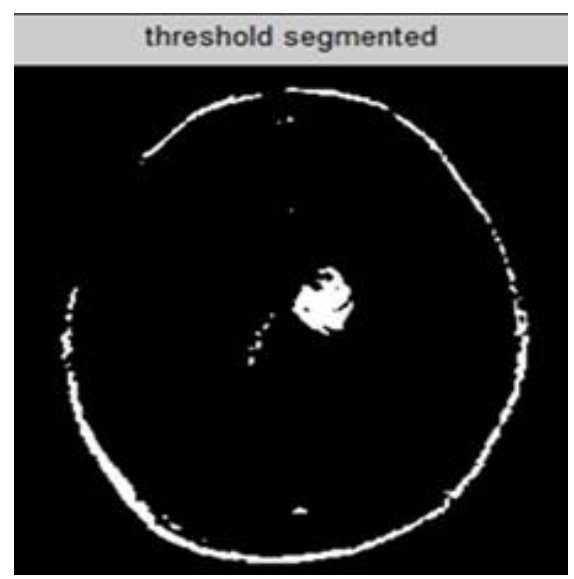

Fig 3: Image after Thresholding

\subsection{Binarization and line segmentation Sauvola} algorithm

Sauvola Algorithm is a thresholding technique algorithm where thresholds are local this technique gives very good results when the background of the image is non- uniform.

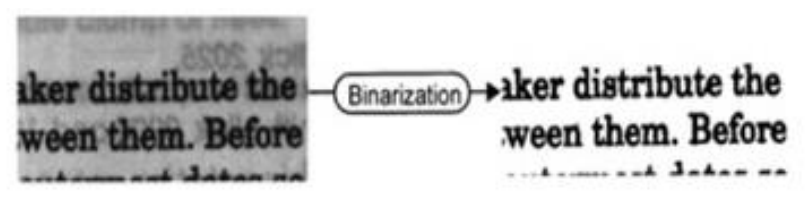

$$
T(x, y)=m(x, y) \cdot\left\lfloor 1+k \cdot\left(\frac{s(x, y)}{R}-1\right)\right\rfloor,
$$

Fig 4: Binarization \& Segmentation

\subsection{Boundary Based Approach}

Pixels are assigned to groups in Thresholding in accordance with the group of values in which a pixel resides. Thresholding is the relatively simple most often used segmentation process. Because of a single threshold, $t$, the lattice location pixel $(i, j)$, with a Gray scale value of fij, is assigned to category 1 if fij $\leq \mathrm{t}$, or else the pixel is assigned to category 2 . This approach is also called as Thresholding.

\subsection{Edge Based Approach}

An edge filter is applied to the image in edge-based segmentation, pixels are classified as edge-pixels or non- 
edge pixels based on the performance of the filter, and pixels which are not separated by an edge are placed into the same category. Edge based image segmentation is based on the assumption that an extreme of the first order derivative or a zero crossing in the second order derivative gives the position of an edge. The pixels are labelled as an object pixel based exclusively on its Gray value, regardless of the frame of reference. Component computation and segmentation can be replicated to maximize the results before the method converges into a coherent outcome.

\subsection{Region-Dependent Approach}

Region-dependent segmentation methods function iteratively by grouping neighboring pixels together and providing identical values and separating pixel categories that are unidentical in value. Spatial clustering can be known as segmentation. Clustering in the sense of clustering together pixels with similar values while the spatial component often forms a single related component in that pixel in the same category.

\subsection{The Convolution Neural Network Algorithm}

Convolution Neural Networks (CNNs) in recent times has been shown to be an efficient class of models for understanding image information, providing state-of-the- art image recognition, segmentation, identification, and recovery outcomes. System for video summarization that uses extracted Video Frames for pre-processing. These featured frames are thereafter analyzed via Convolutional Neural Networks (CNN).

Using this analysis, features are extracted and calculated, which are used for generation of summarized videos. As well as multiple hidden layers, a CNN consists of an input and an output layer. Convolutional layers, pooling layers, completely linked layers and normalization layers are usually the hidden layers of a CNN. CNN will be used to train the data analytics engine for recognizing disease from the image.

Accepts a volume of size W1 x H1 x D1.

Requires four hyper parameters:

Number of filters K.

Their spatial extent F.

The stride $\mathrm{S}$.

The amount of zero padding $\mathrm{P}$.

Produces a volume of size $\mathrm{W} 2$ x H2 x D2 where:

$\mathrm{W} 2=(\mathrm{W} 1-\mathrm{F}+2 \mathrm{P}) / \mathrm{S}+1$.

$\mathrm{H} 2=(\mathrm{H} 1-\mathrm{F}+2 \mathrm{P}) / \mathrm{S}+1$ (i.e., width and height are computed equally by symmetry).

D2 $=\mathrm{K}$

With parameter sharing it introduces F x F x D1 weights per filter, for a total of (F x F x D1) x K weights and $\mathrm{K}$ biases. (i.e., width and height are computed equally by symmetry).

In the output volume, the dth depth slice (of size W2 x H2) is the result of performing a valid convolution of the dth filter over the input volume with a stride of $S$, and then offset by dth bias.

A common setting of the hyper parameters is $\mathrm{F}=3, \mathrm{~S}=1, \mathrm{P}$ $=1$.

\subsection{K-means clustering}

It is an iterative and evolutionary method that seeks to split the dataset into different nonoverlapping datasets sub category (clusters) identified by $\mathrm{K}$ pre-defined, where a single category corresponds to every other data point. This attempts to keep the data points of the same category(cluster) as close as feasible while still keeping the data points of the different categories(clusters) as distinct (far) as viable ${ }^{[5]}$. It assigns the data points of a cluster in such a way that the sum of the square distance between the data points and the middle of the cluster (the arithmetical mean across all data points that belong to the cluster) is at least the same.

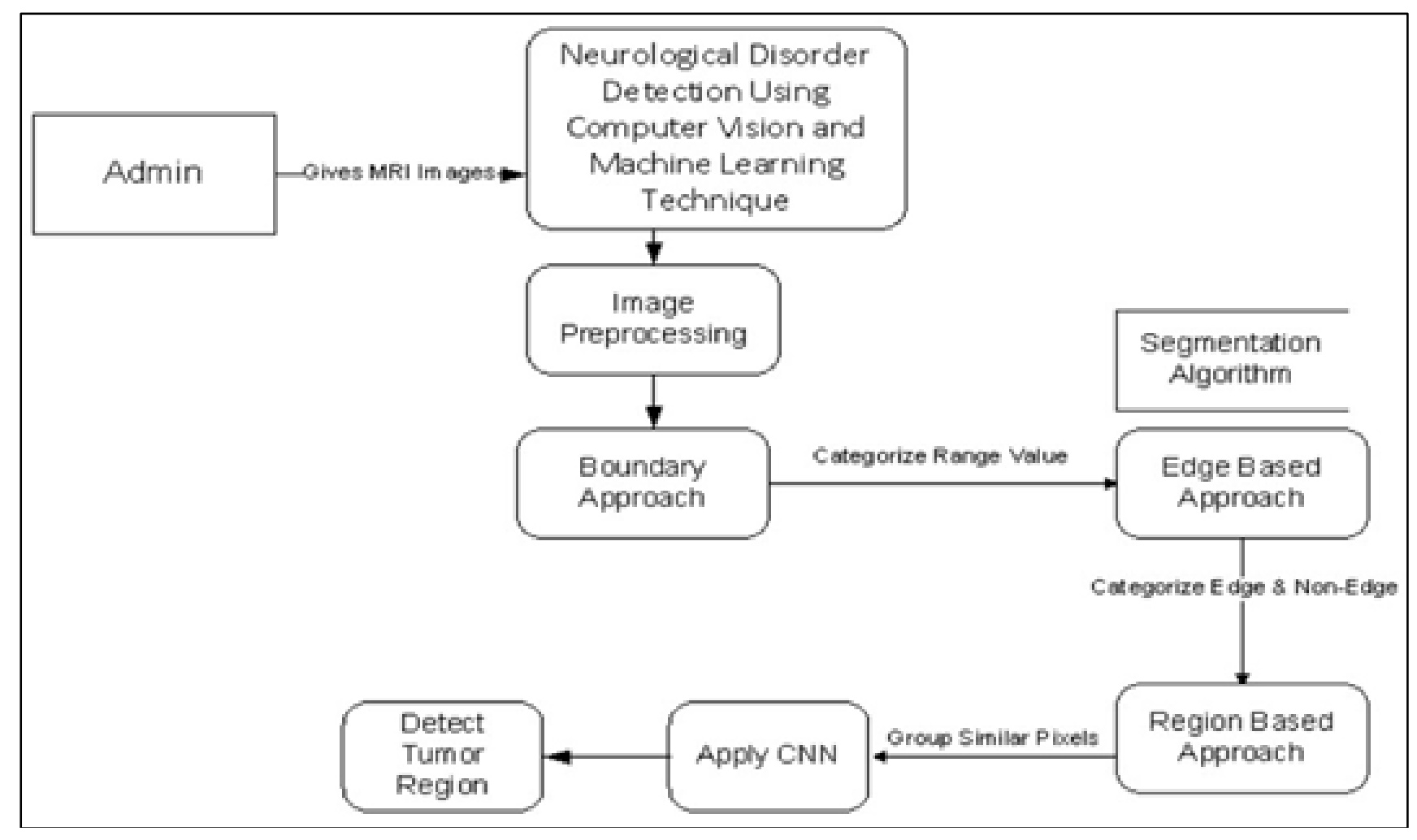

Fig 5: Data Flow Diagram 


\section{Results}

We applied pre-processing on input image (OpenCV image) and got the desired outcome, edge detection grey scale conversion was done successfully. Pixels are categorized as edge or non-edge depending on the filter output using edgebased segmentation on image. In region-based segmentation, regional maxima were applied to the image for grouping the pixels which are neighbors and have similar values. The training and testing of CNN model was carried out followed by the application of K-means algorithm to find the region of interest (ROI) which was then analyzed for identification of tumor.

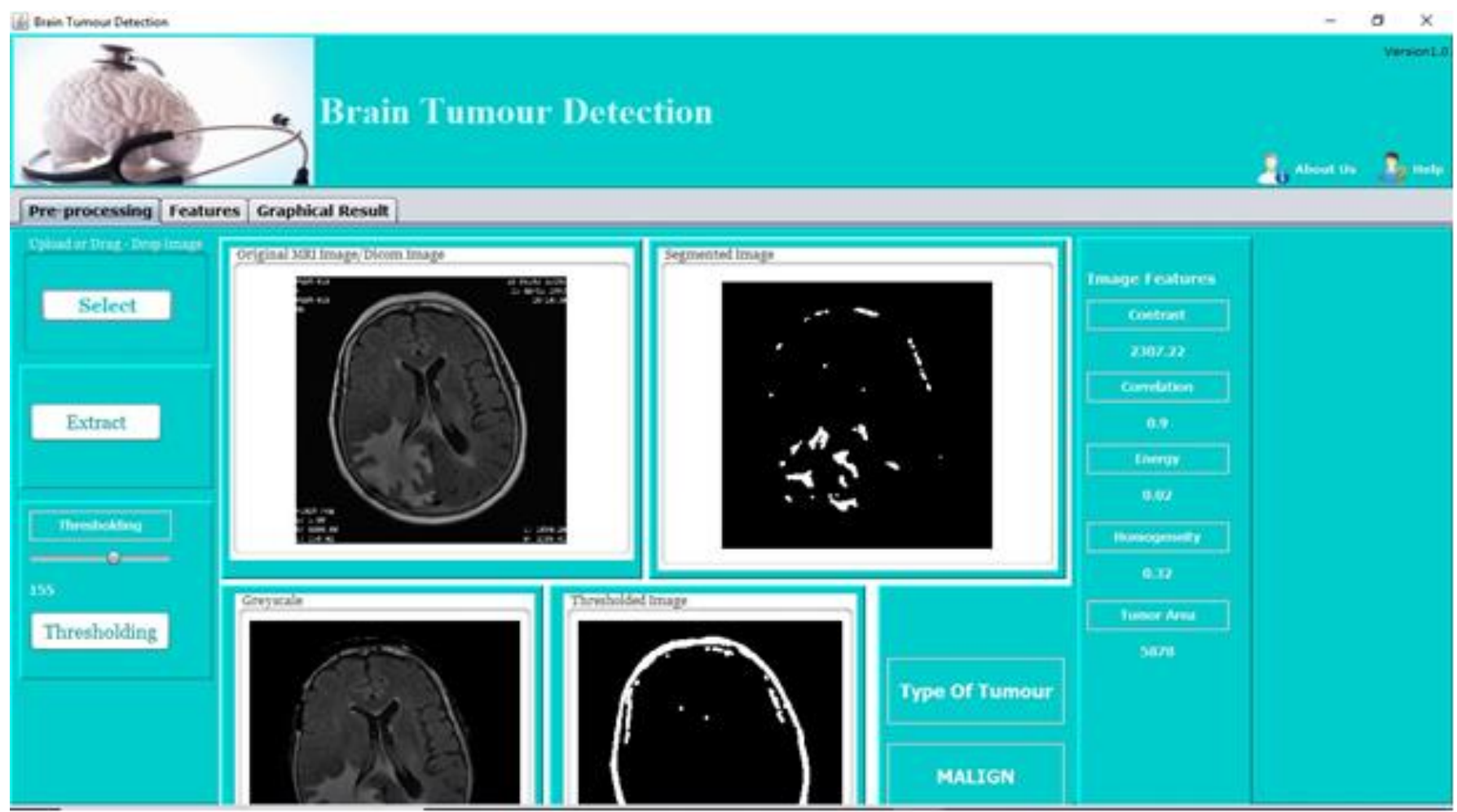

Fig 6: MRI Image Pre-Processing

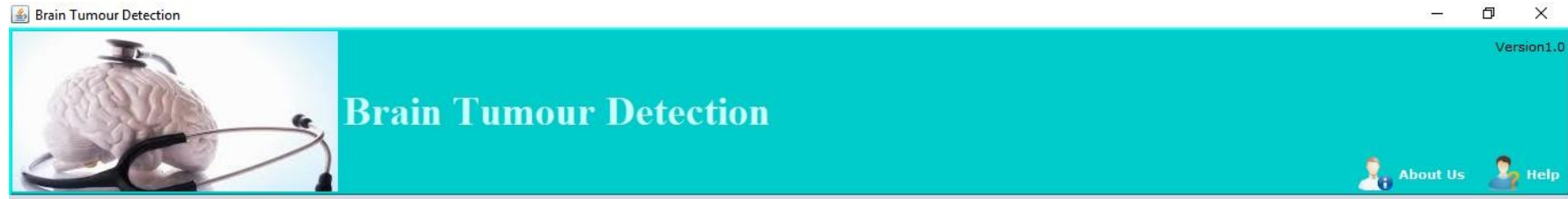

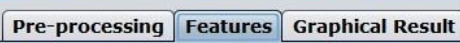

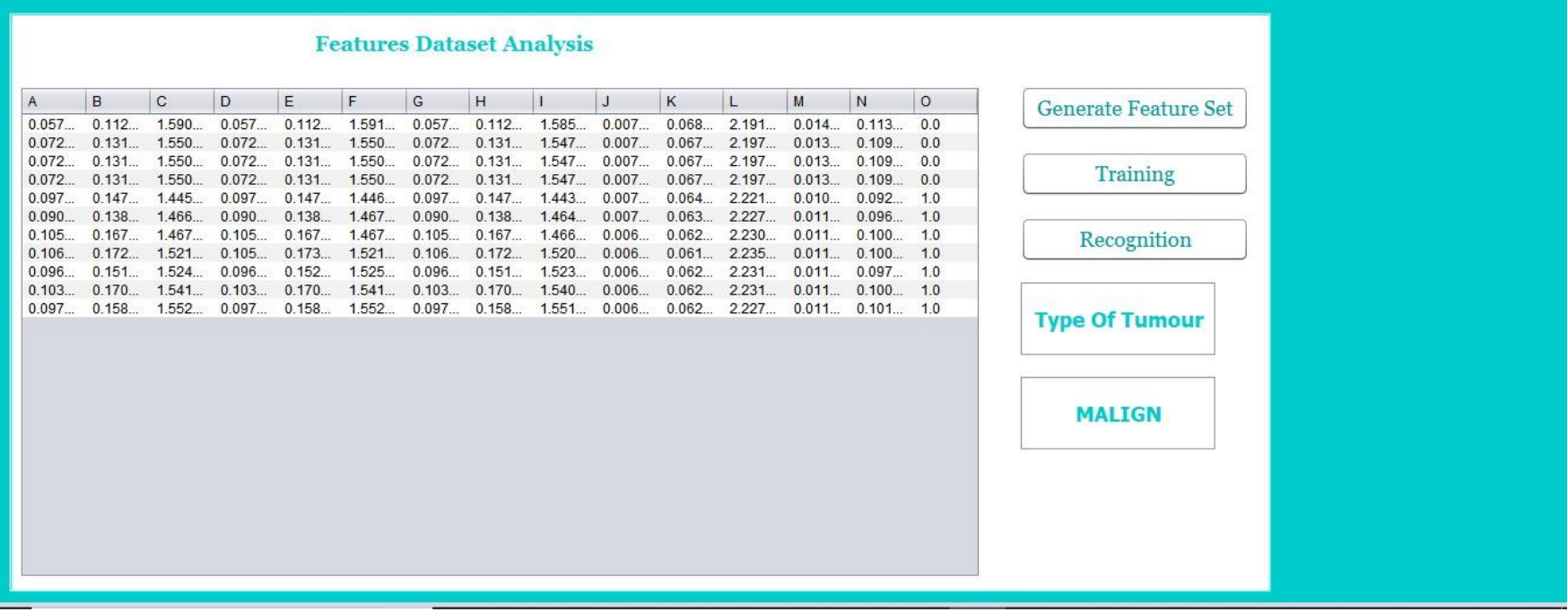

Fig 7: Featured Analysis 


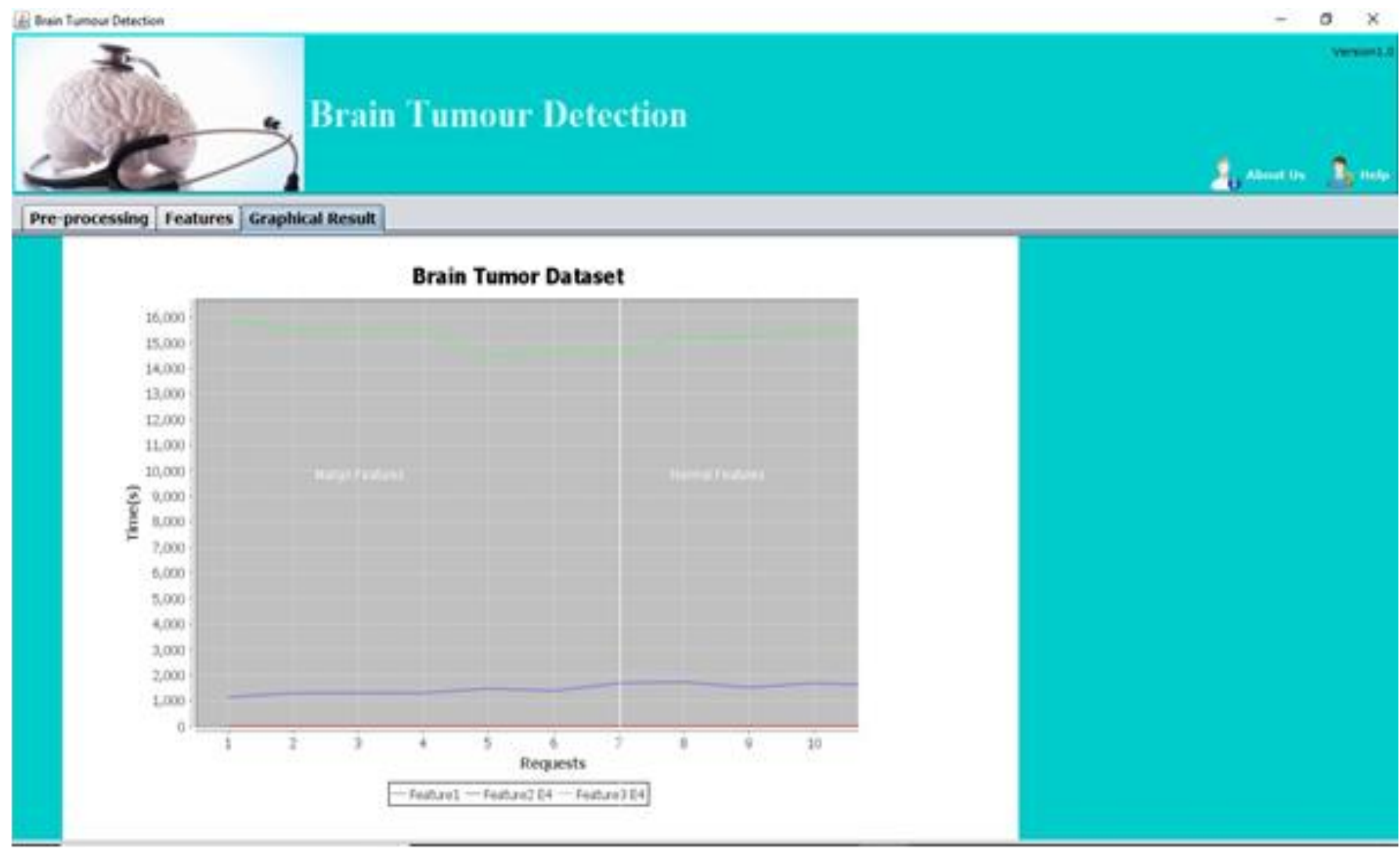

Fig 8: Result: Normal or Malign

\subsection{Data Flow Analysis}

\section{- $\quad$ Event 1}

User will input the MRI image to the SYSTEM. Let $\mathrm{f}(\mathrm{U})$ be a function of User Thus, $\mathrm{f}(\mathrm{U})\{\mathrm{S}\}$

\section{- $\quad$ Event 2}

Pre-Processing is performed on input images. Let $\mathrm{f}(\mathrm{S})$ be an image processing function of the system. $\mathrm{P}=$ Preprocessing Thus, $\mathrm{f}(\mathrm{S}) \rightarrow\{\mathrm{I} 1, \mathrm{I} 2, \mathrm{I} 3 \ldots \mathrm{In}\} \& \mathrm{P}$

\section{- $\quad$ Event 3}

Apply Edge and Region based Segmentation on image Let $\mathrm{f}(\mathrm{S})$ be system function.

Thus, $\mathrm{f}(\mathrm{S}) \longrightarrow\{\mathrm{E} \mathrm{U} \mathrm{R}\} \varepsilon\{\mathrm{I} 1, \mathrm{I} 2, \mathrm{I} 3 \ldots . \mathrm{In}\}$

\section{- $\quad$ Event 4}

Let $\mathrm{f}(\mathrm{FT})$ is a function used to extract features.

Thus, $\mathrm{f}(\mathrm{FT}) \rightarrow\{\mathrm{I} 1, \mathrm{I} 2, \mathrm{I} 3 \ldots . \mathrm{In}\} \varepsilon$

$\{\mathrm{F} 1, \mathrm{~F} 2, \mathrm{~F} 3 \ldots . . \mathrm{Fn}\}$.

\section{- $\quad$ Event 5}

Let $\mathrm{f}(\mathrm{AC})$ is a tumor identification function of the system.

Using $\mathrm{CNN}$ system can detect tumor.

Thus, $\mathrm{f}(\mathrm{AC})$ ! $\{\mathrm{F} 1, \mathrm{~F} 2, \mathrm{~F} 3 \ldots \mathrm{Fn}\} \varepsilon \mathrm{T}$.

\subsection{Mathematical Model}

Let us consider $\mathrm{S}$ be a Systems such that $\mathrm{S}=\mathrm{U}, \mathrm{ES}, \mathrm{SS}, \mathrm{K}$, DE, DS;

- $\mathrm{U}=\{\mathrm{U} 1, \mathrm{U} 2, \mathrm{U} 3 \ldots . . . . \mathrm{Un} \mathrm{j}$ ' $\mathrm{U}$ ' is a Set of all USERS $\} \mathrm{U}$ is the users of the system. Users of the system may grow as the system is used by more and more people. User is infinite set.

- $\quad I=\{I 1, I 2, I 3 \ldots$. In $\mid I$ are the input images $\} \mathrm{I}$ is images used in the system. These images may increase day by day. This is infinite set.
- $\quad \mathrm{FT}=\{\mathrm{F} 1, \mathrm{~F} 2, \mathrm{~F} 3 \ldots . \mathrm{Fn} \mid \mathrm{F}$ are the features extracted $\} \mathrm{F}$ is the features extracted from pre-processed image in the system.

- $\mathrm{T}=\{\mid \mathrm{T}$ is the technique used to process Input image $\} \mathrm{T}$ is the technique used for image processing. This is a finite set.

- $\quad \mathrm{AC}=\{\mathrm{AC}$ is the classification action taken by system during $\} \mathrm{AC}$ is the classification action performed by the system.

- $\quad \mathrm{DS}=\{$ User_Info, Train_Data, $\mid \mathrm{DS}$ is a Set of data table for permanent storing of data on server $\}$

- $\quad S S=\left\{S_{-}\right.$images, $S_{-}$Features $\mid$SS is a Set of Storage Service\} Storage Server will provide services for storing images and emotion detected. As this set also has finite attributes, so this is also Finite Set.

\section{Conclusion}

Here an algorithm like CNN-based segmentation methods has been developed for the detection of brain tumor from MRI brain images by performing different operations like Edge Detection, Thresholding followed by segmentation. Here we are using two types of segmentation methods like edge-based segmentation and Region-based segmentation to segment or partition the digital image into multiple segments of pixels. By applying neural network algorithm for training with balanced classes and then refining it with proportions near the originals binary $\mathrm{CNN}$ to identify the complete tumor.

In the future with improved algorithms more accuracy can be achieved in the results of detecting the tumors' size and stage of the cancer. This project can also be extended into finding other organ related diseases. With more data available in the future this project can be helpful for animals as well. 


\section{References}

1. Pranav Shetty, Suraj Singh, Rasvi Jambhulkar, Kajal Sheth, Deepali Ujalambkar. A survey on detection of brain tumor using computer vision and machine learning technique, International Journal of Applied Research 2021.

2. Arnaud A, Forbes F, Coquery N, Collomb N, Lemasson B, Barbier EL. Fully Automatic Lesion Localization and Characterization: Application to Brain Tumors using Multi parametric Quantitative MRI Data, Transactions on Medical Imaging.

3. Pereira S, Pinto A, Alves V, Silva CA. Brain Tumor Segmentation using Convolutional Neural Networks in MRI Images, IEEE Transactions on Medical Imaging 2016.

4. Hema Malhotra, Sameena Naaz. Analysis of MRI images using data mining for detection of brain tumor. IJARCS 2018.

5. Pranav Shetty, Suraj Singh. Hierarchical Clustering: A Survey, International Journal of Applied Research 2021.

6. Pallavi Shrivastava, Akhilesh Upadhayay, Akhil Khare. Devising Classifiers for Analyzing and Classifying Brain Tumor using Integrated Framework PNN, International Conference on Energy Systems and Applications 2015.

7. Nalbalwar R, Majhi U, Patil R, Gonge S. Detection of Brain Tumor by using ANN. International Journal of Research in Advent Technology 2014;2:4.

8. Joseph RP, Singh CS, Manikandan M. Brain tumor MRI image segmentation and detection in image processing. International Journal of Research in Engineering and Technology 2014.

9. Salunkhe PB, Patil PS, Bhamare DR. Brain Tumor Detection and Area Calculation of Tumor in Brain MRI mages using Clustering Algorithms, IOSR - JECE).

10. Samriti, Mr. Paramveer Singh. Brain Tumor Detection Using Image Segmentation. International Journal of Engineering Development and Research 2016.

11. Sonu Suhag, Saini LM. Automatic Detec Tion of Brain Tumor by Image Processing in Matlab. International Journal of Advances in Science Engineering and Technology 2015.

12. Shelkar R, Thakare MN. Brain Tumor Detection and Segmentation by using Thresholding and Watershed Algorithm. IJACT 2014, 1(3). 